\title{
Model Predictive Control for Linear Complementarity and Extended Linear Complementarity Systems
}

\author{
Bambang Riyanto ${ }^{*}$ \& Ibrahim Hakim* \\ ${ }^{*}$ Department of Electrical Engineering \\ Institut Teknologi Bandung, \\ Jl. Ganesha 10, Bandung, Indonesia \\ briyanto@1skk.ee.itb.ac.id
}

\begin{abstract}
In this paper, we propose model predictive control method for linear complementarity and extended linear complementarity systems by formulating optimization along prediction horizon as mixed integer quadratic program. Such systems contain interaction between continuous dynamics and discrete event systems, and therefore, can be categorized as hybrid systems. As linear complementarity and extended linear complementarity systems finds applications in different research areas, such as impact mechanical systems, traffic control and process control, this work will contribute to the development of control design method for those areas as well, as shown by three given examples.
\end{abstract}

Keywords: model predictive control; linear complementarity; extended linear complementarity; hybrid systems; mixed integer quadratic program; impact mechanical systems; traffic control; process control.

\section{$1 \quad$ Introduction}

Many engineering systems in real world do not only contain continuous dynamics (i.e. systems that can be described by differential or difference equations), but also interaction between continuous and discrete event dynamics (i.e. asynchronous systems where the state transitions are initiated by events). This kind of systems can be categorized as hybrid systems, which is a subject of very active research area in the last few years. Hybrid systems arise throughout industry such as traffic control, robot design, flight control, process control and path planning. Several modeling frameworks have been developed for hybrid systems. See $[13,15]$ for the survey on modeling, analysis and control design for hybrid systems.

Linear complementarity (LC) system is one of the restricted model for hybrid systems. This model finds application in modeling of mechanical systems with impact such as found in manipulator trajectory on constrained environment and bipedal robot [4]. This model also finds application in electronics circuit containing discontinuous element such as diode and transistor [10]. From 
theoretical point of view, well-posedness issue and properties of such systems were studied in [17]. See [4] for the survey of recent results on LC systems. Extension of LC systems is known as Extended Linear Complementarity (ELC) systems, and was studied in [5-8] for dynamic traffic light control systems in single or multi-road intersection. Optimal control scheme has been developed for these systems using tools from mathematical problem: extended linear complementarity problem (ELCP).

Most of previous results in this area focused on system analysis[13], and very few results addressed control synthesis. Moreover, extension of synthesis results of the linear time invariant systems to LC systems is not straight forward due to the intrinsically complex structure of LC systems. In this paper, we propose a model predictive control method for LC and ELC systems.

This paper is organized as follows. In Section 2, some preliminaries for LC and ELC systems are presented. In Section 3, a solution to quadratic optimization problem is derived for LC and ELC systems. In Section 4, optimal control method is developed for LC systems. In Section 5, similar optimal control method is presented for ELC systems. Section 6 addresses model predictive control for both LC and ELC systems. Numerical examples are presented in Section 7. Finally, conclusion is drawn in Section 8.

\section{Preliminaries}

In this section, some preliminaries for LC and ELC systems are presented.

Definition 1 - linear complementarity systems: In discrete time, linear complementarity (LC) systems are given by the equations:

$$
\begin{aligned}
& x(k+1)=A x(k)+B_{1} u(k)+B_{2} w(k) \\
& y(k)=C x(k)+D_{1} u(k)+D_{2} w(k) \\
& v(k)=E_{1} x(k)+E_{2} u(k)+E_{3} w(k)+g_{4} \\
& 0 \leq v(k) \perp w(k) \geq 0
\end{aligned}
$$

where $\quad x(k) \in R^{n}, \quad u(k) \in R^{m}, \quad y(k) \in R^{l} \quad$ are state, input, output, $v(k), w(k) \in R^{s}$ are complementarity variables, $0 \leq v(k) \perp w(k) \geq 0$ means $v(k)=0, w(k) \geq 0$ or $v(k) \geq 0, w(k)=0, A, B_{1}, B_{2}, C, D_{1}, D_{2}, E_{1}, E_{2}$, $E_{3}$ and $g_{4}$ are constant matrices with suitable dimensions. 
Definition 2 - extended linear complementarity systems: In discrete time, extended linear complementarity systems are given by

$$
\begin{aligned}
& x(k+1)=A x(k)+B_{1} u(k)+B_{2} d(k) \\
& y(k)=C x(k)+D_{1} u(k)+D_{2} d(k) \\
& E_{1} x(k)+E_{2} u(k)+E_{3} d(k) \leq g_{4} \\
& \sum_{i=1}^{p} \prod_{j \in \phi_{i}}\left(g_{4}-E_{1} x(k)-E_{2} u(k)-E_{3} d(k)\right)_{j}=0
\end{aligned}
$$

where $x(k) \in R^{n}, u(k) \in R^{m}, y(k) \in R^{l}, d(k) \in R^{r}$ are state, input, output and auxiliary variable, respectively. $A, B_{1}, B_{2}, C, D_{1}, D_{2}, E_{1}, E_{2}, E_{3}$ and $g_{4}$ are constant matrices with suitable dimension. If $g_{4} \in R^{q \times 1}$ then $p$ is a number of $\phi_{1}, \phi_{2}, \ldots, \phi_{p} \subseteq\{1, \ldots, q\}$.

Two types of optimization problems will be used extensively in this paper: mixed integer feasibility problem and mixed integer quadratic program.

Definition 3 - mixed integer linear feasibility problem (MILFP): Given $A \in R^{p \times n}$ and $b \in R^{p}, n_{c}$ is dimension of real state variables and $n_{d}$ is dimension of integer state variables, find $x \in R^{n c} \times\{0,1\}^{\text {nd }}$ such that $A x \leq b$.

Definition 4 - mixed integer quadratic problem (MIQP): Given $A \in R^{p \times n}$ and $b \in R^{p}$, find $x \in R^{n c} \times\{0,1\}^{\text {nd }}$ that solved the optimization problem

$$
\min _{x}\left(f^{T} x\right)
$$

subject to: $A x \leq b$

Remark 1: MILFP and MIQP can be solved using branch and bound method [2], and a MATLAB program has also been developed to compute their solutions numerically.

In the next section, linear complementarity and extended linear complementarity problems are used to develop control method for LC and ELC systems, respectively. 
Definition 4 - linear complementarity problem (LCP): Given $M \in R^{n \times n}$ and $q \in R^{n \times 1}$, find $w, z \in R^{n}$ such that

$$
\begin{aligned}
& w=M z+q \\
& 0 \leq w \perp z \geq 0
\end{aligned}
$$

where $0 \leq w \perp z \geq 0$ means $w=0, z \geq 0$ or $w \geq 0, z=0$.

Remark 2: Several algorithms were proposed to solve LCP. One approach is based on Lemke's method [1]. However, this approach extensively uses tableau and pivot operations which are not efficient for high dimension problem. Another approach is based on convex optimization [9], and resulted in a MATLAB program to compute LCP solution numerically.

Remark 3: Based on the idea of [8] and [11], LCP can be cast as MILFP. Assign logical variable $\delta_{i} \in\{0,1\}, i=1, \ldots, n$, which corresponds to each complementarity term $0 \leq w_{i} \perp z_{i} \geq 0$ as

$$
\begin{aligned}
& \left(w_{i}=0\right) \leftrightarrow\left(\delta_{i}=1\right), z_{i} \geq 0 \\
& \left(z_{i}=0\right) \leftrightarrow\left(\delta_{i}=0\right), w_{i} \geq 0
\end{aligned}
$$

As in [3], this relation can be transformed in to mixed integer linear inequalities

$$
\begin{aligned}
& w_{i} \leq M_{w i}\left(1-\delta_{i}\right), z_{i} \geq 0 \\
& z_{i} \leq M_{z i} \delta_{i}, w_{i} \geq 0
\end{aligned}
$$

where $M_{w i}=\max \left(w_{i}\right)$ and $M_{z i}=\max \left(z_{i}\right)$. By collecting the above inequalities for $i=1 . . . n$, the following inequalities hold

$$
\begin{aligned}
& w \leq M_{w}(I-\delta), z \geq 0 \\
& z \leq M_{z} \delta, w \geq 0
\end{aligned}
$$

By substituting equation (9) into (11) and (12), we get MILFP

$$
\left[\begin{array}{cc}
M & M_{w} \\
I & -M_{z} \\
-M & 0 \\
-I & 0
\end{array}\right]\left[\begin{array}{l}
z \\
\delta
\end{array}\right] \leq\left[\begin{array}{c}
M_{w} I-q \\
0 \\
q \\
0
\end{array}\right]
$$


Definition 5 - extended linear complementarity problem (ELCP): Given $A \in R^{p \times n}, c \in R^{p \times 1}$ and $m$ index set $\phi_{1}, \phi_{2}, \ldots, \phi_{m} \subseteq\{1, \ldots, p\}$, find $x \in R^{n}$ such that

$$
\begin{aligned}
& A x \geq c \\
& \sum_{j=1}^{m} \prod_{i \in \phi_{j}}(A x-c)_{i}=0
\end{aligned}
$$

Remark 4: ELCP can be solved numerically using algorithm proposed in [5]. This algorithm gives complete solution in the form of feasible polyhedral region on the solution space.

Remark 5: In [8], it was shown that ELCP can be cast as MILFP, by assigning logical variables $\delta_{i} \in\{0,1\}, i=1, \ldots, n$, which corresponds to each extended linear complementarity term as $\left((A x-c)_{i} \geq 0\right) \leftrightarrow\left(\delta_{i}=1\right)$.

As in [3], this relation can be transformed into mixed integer linear inequalities

$$
(A x-c)_{i} \leq d_{i i}^{u p p} \delta_{i}
$$

where $d_{i i}^{u p p}=\max (A x-c)_{i}$, upp denotes upper bound of $(A x-c)_{i}$ term. By collecting the above inequalities for $i=1 \ldots p$ we get

$$
A x-c \leq \underbrace{\left[\begin{array}{ccc}
d_{11}^{u p p} & \cdots & 0 \\
\vdots & \ddots & \vdots \\
0 & \cdots & d_{p p}^{u p p}
\end{array}\right]}_{D_{u p p}} \underbrace{\left[\begin{array}{c}
\delta_{1} \\
\vdots \\
\delta_{p}
\end{array}\right]}_{\delta}
$$

Extended complementarity condition (14) implies that at least one inequality should hold with equalities. This can be represented by inequalities

$$
\sum_{i \in \phi_{j}} \delta_{i} \leq \# \phi_{j}-1
$$

where $\# \phi_{j}$ is cardinality of $\phi_{j}$. By collecting the above inequality for $j=1 \ldots m$, the following inequalities hold 


$$
\underbrace{\left[\begin{array}{ccc}
s_{11} & \cdots & s_{1 p} \\
\vdots & \ddots & \vdots \\
s_{m 1} & \cdots & s_{m p}
\end{array}\right]}_{S} \underbrace{\left[\begin{array}{c}
\delta_{1} \\
\vdots \\
\delta_{p}
\end{array}\right]}_{\delta} \leq \underbrace{\left[\begin{array}{c}
\# \phi_{1}-1 \\
\vdots \\
\# \phi_{m}-1
\end{array}\right]}_{t}
$$

where $s_{j i}=0$ if $i \notin \phi_{j}$, and $s_{j i}=1$ if $i \in \phi_{j}$. By collecting (13),(15) and (16), we get MILFP

$$
\left[\begin{array}{cc}
-A & 0 \\
A & -D_{u p p} \\
0 & S
\end{array}\right]\left[\begin{array}{l}
x \\
\delta
\end{array}\right] \leq\left[\begin{array}{c}
-c \\
c \\
t
\end{array}\right]
$$

\section{Solution of quadratic optimization subject to LCP and ELCP}

In the following discussion, definitions of quadratic optimization problem subject to LCP and ELCP are used to develop model predictive control method for LC and ELC systems, respectively. We propose solution for these problems by formulating them as MIQP.

Problem 1 - quadratic optimization problem subject to LCP: Find solution $x \in R^{n}$ for the optimization problem

$$
\begin{aligned}
& \min \left(\frac{1}{2} x^{T} Q x+f^{T} x\right) \\
& \text { subject to: } x^{T}(M x+q)=0 \\
& M x+q \geq 0, x \geq 0
\end{aligned}
$$

Solution: From Remark 3, the LCP (18) and (19) can be converted into MILFP

$$
\underbrace{\left[\begin{array}{cc}
M & M_{w} \\
I & -M_{z} \\
-M & 0 \\
-I & 0
\end{array}\right]}_{\bar{A}} \underbrace{\left[\begin{array}{c}
x \\
\delta
\end{array}\right]}_{v} \leq \underbrace{\left[\begin{array}{c}
M_{w} I-q \\
0 \\
q \\
0
\end{array}\right]}_{\bar{b}} .
$$

The cost function can be modified into 


$$
J=\frac{1}{2} x^{T} Q x+f^{T} x=\frac{1}{2} \underbrace{\left[\begin{array}{c}
x \\
\delta
\end{array}\right]^{T}}_{v^{T}} \underbrace{\left[\begin{array}{ll}
Q & 0 \\
0 & 0
\end{array}\right]}_{H} \underbrace{\left[\begin{array}{c}
x \\
\delta
\end{array}\right]}_{v}+\underbrace{\left[\begin{array}{c}
f \\
0
\end{array}\right]^{T}}_{\vec{f}} \underbrace{\left[\begin{array}{c}
x \\
\delta
\end{array}\right]}_{v}
$$

Therefore, the optimization problem can now be formulated into MIQP as follows

$$
\begin{aligned}
& \min _{v}\left(\frac{1}{2} v^{T} H v+\bar{f}^{T} v\right) \\
& \text { subject to: } \bar{A} v \leq \bar{b} \\
& v_{i} \in\{0,1\}, i \in\{n+1, \ldots, 2 n\}
\end{aligned}
$$

Problem 2 - quadratic optimization problem subject to ELCP: Find solution $x \in R^{n}$ for the optimization problem

$$
\min \left(\frac{1}{2} x^{T} Q x+f^{T} x\right)
$$

subject to: $A x \geq c$

$$
\sum_{j=1}^{m} \prod_{i \in \phi_{j}}(A x-c)_{i}=0
$$

Solution: From Remark 5, ELCP (21) and (22) can be converted into MILFP

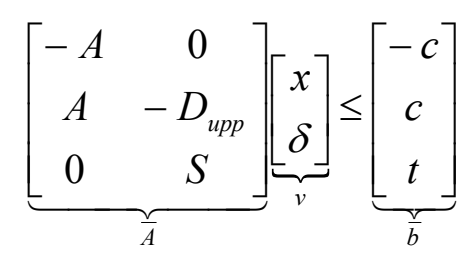

The cost function can be modified into

$$
J=\frac{1}{2} x^{T} Q x+f^{T} x=\frac{1}{2} \underbrace{\left[\begin{array}{c}
x \\
\delta
\end{array}\right]^{T}}_{v^{T}} \underbrace{\left[\begin{array}{ll}
Q & 0 \\
0 & 0
\end{array}\right]}_{H} \underbrace{\left[\begin{array}{c}
x \\
\delta
\end{array}\right]}_{v}+\underbrace{\left[\begin{array}{c}
f \\
0
\end{array}\right]^{T}}_{\vec{f}} \underbrace{\left[\begin{array}{c}
x \\
\delta
\end{array}\right]}_{v}
$$

Therefore, the optimization problem can now be recast as MIQP 


$$
\begin{aligned}
& \min _{v}\left(\frac{1}{2} v^{T} H v+\bar{f}^{T} v\right) \\
& \text { subject to: } \bar{A} v \leq \bar{b} \\
& v_{i} \in\{0,1\}, i \in\{n+1, \ldots, n+p\}
\end{aligned}
$$

\section{Optimal Control Problem for LC Systems}

Consider optimal control problem for LC systems: find sequence of $u(0), u(1), \ldots, u(N-1)$ that minimize the cost function

$$
J=\sum_{k=0}^{N-1}\left(x(k)-x_{r e f}\right)^{T} Q\left(x(k)-x_{r e f}\right)+u(k)^{T} R u(k)
$$

subject to LC dynamics (1)-(4).

Proposition 1: Quadratic optimal control of LC system can be written as quadratic optimization subject to LCP (Problem 1).

Proof: Given $x(0)$, the state equation can be written recursively

$$
x(k)=A^{k} x(0)+\sum_{i=0}^{k-1} A^{i}\left(B_{1} u(k-1-i)+B_{2} w(k-1-i)\right)
$$

Collect the above equation for $k=0,1, \ldots, N-1$ to

$$
X=\bar{A} x(0)+\bar{B}_{1} U+\bar{B}_{2} W
$$

where $X=[x(0) \ldots x(N-1)]^{T}, U=[u(0) \ldots u(N-1)]^{T}$ and $W=[w(0) \ldots w(N-1)]^{T}$. The idea is to consider $U$ as an unknown complementarity variable. Since complementarity variable is always bigger than or equal to zero, we separate $U$ as positive and negative components as $U=U^{+}-U^{-}$and $0 \leq U^{+} \perp U^{-} \geq 0$. By defining $Z=\left[\begin{array}{lll}U^{+} & U^{-} & W\end{array}\right]^{T}$ we can rewrite equation (25) as

$$
X=\bar{A} x(0)+\left[\begin{array}{lll}
\bar{B}_{1} & -\bar{B}_{1} & \bar{B}_{2}
\end{array}\right] Z
$$

Substitute equation (24) into (3), and write recursively as

$$
V=\bar{E}_{1} x(0)+\bar{G}_{4}+\left[\begin{array}{lll}
\bar{E}_{2} & -\bar{E}_{2} & \bar{E}_{3}
\end{array}\right] Z
$$


Introduce auxiliary complementarity variable $V_{2}=U^{-}$and $V_{3}=U^{+}$to result in auxiliary complementarity term $0 \leq U^{+} \perp V_{2} \geq 0$ and $0 \leq U^{-} \perp V_{3} \geq 0$. By writing $Y=\left[\begin{array}{lll}V_{2} & V_{3} & V\end{array}\right]^{T}$ and substituting equation (26) into cost function (23), we obtain quadratic optimization problem subject to LCP (Problem 1):

$$
\begin{aligned}
& \min _{Z}\left(Z^{T} H Z+f^{T} Z\right) \\
& \text { subject to: } Y=\left[\begin{array}{c}
0 \\
0 \\
\bar{E}_{1} x(0)+\bar{G}_{4}
\end{array}\right] x(0)+\left[\begin{array}{ccc}
0 & I & 0 \\
I & 0 & 0 \\
\bar{E}_{2} & -\bar{E}_{2} & \bar{E}_{3}
\end{array}\right] Z
\end{aligned}
$$$$
0 \leq Y \perp Z \geq 0
$$

Remark 6: By using solution of Problem 1, we can formulate quadratic optimization subject to LCP as MIQP that can be solved numerically using available program [2].

Remark 7: We can also formulate quadratic optimal control problem into MIQP directly. Substitute equation (24) to (3) to get

$$
V=\bar{E}_{1} x(0)+\bar{E}_{2} U+\bar{E}_{3} W+\bar{G}_{4}
$$

Then, the complementarity term can be described by

$$
0 \leq V \perp W \geq 0
$$

Equation (29) can be further transformed into mixed integer linear inequalities

$$
\begin{aligned}
& V \leq M_{V}(I-\delta), W \geq 0 \\
& W \leq M_{W} \delta, V \geq 0
\end{aligned}
$$

By substituting equation (28) to (30),(31), and introducing $Z=\left[\begin{array}{lll}U & W & \delta\end{array}\right]^{T}$ then

$$
\left[\begin{array}{ccc}
\bar{E}_{2} & \bar{E}_{2} & M_{V} \\
0 & I & -M_{W} \\
-\bar{E}_{2} & -\bar{E}_{3} & 0 \\
0 & -I & 0
\end{array}\right] Z \leq\left[\begin{array}{c}
M_{V}-\bar{E}_{1} x(0)-\bar{G}_{4} \\
0 \\
\bar{E}_{1} x(0)+\bar{G}_{4} \\
0
\end{array}\right]
$$

Notice that equation (25) can be written as

$$
X=\bar{A} x(0)+\left[\begin{array}{lll}
\bar{B}_{1} & \bar{B}_{2} & 0
\end{array}\right] Z
$$


Substituting Equation (33) into cost function (23) results in MIQP:

$$
\min _{Z}\left(Z^{T} H Z+f^{T} Z\right)
$$

subject to (32)

\section{Optimal Control Problem for ELC Systems}

Consider optimal control problems for LC systems: find sequence of $u(0), u(1), \ldots, u(N-1)$ that minimize the cost function

$$
J=\sum_{k=0}^{N-1}\left(x(k)-x_{r e f}\right)^{T} Q\left(x(k)-x_{r e f}\right)+u(k)^{T} R u(k)
$$

subject to ELC dynamics (5)-(8).

Proposition 2: Quadratic optimal control of ELC system can be recast as quadratic optimization subject to ELCP (Problem 2).

Proof: Given $x(0)$, state equation can be written recursively as

$$
x(k)=A^{k} x(0)+\sum_{i=0}^{k-1} A^{i}\left(B_{1} u(k-1-i)+B_{2} d(k-1-i)\right)
$$

Collect this equation for $k=0,1, \ldots, N-1$ as

$$
X=\bar{A} x(0)+\bar{B}_{1} U+\bar{B}_{2} D
$$

where $\quad X=[x(0) \ldots x(N-1)]^{T}, \quad U=[u(0) \ldots u(N-1)]^{T} \quad$ and $\quad D=[d(0) \ldots d(N-1)]^{T} . \quad$ By introducing $V=\left[\begin{array}{ll}U & D\end{array}\right]^{T}$, Equation (36) can be written as

$$
X=\bar{A} x(0)+\left[\begin{array}{ll}
\bar{B}_{1} & \bar{B}_{2}
\end{array}\right] V
$$

Substitute Equation (35) into Equation (7) to get

$$
\bar{E}_{1} x(0)+\left[\begin{array}{ll}
\bar{E}_{2} & \bar{E}_{3}
\end{array}\right] V \leq \bar{G}_{4}
$$

It follows that the complementarity term (8) is given by

$$
\sum_{i=1}^{(N-1) p} \prod_{j \in \Phi_{i}}\left(\bar{G}_{4}-\bar{E}_{1} x(0)-\left[\bar{E}_{2} \quad \bar{E}_{3}\right] V\right)_{j}=0
$$

where, for $s=1 \ldots N-1, w=1 \ldots p, i=(s-1) p+w, \Phi_{i}()=.\phi_{w}()+.(s-1) q$. 
By substituting equation (37) into cost function (34), we obtain quadratic optimization problem subject to ELCP:

$$
\min _{V}\left(V^{T} H V+f^{T} V\right)
$$

subject to ELCP (38)-(39)

Remark 8: By using solution to Problem 2, we can formulate the quadratic optimization subject to ELCP above as MIQP, which, in turn, can be solved numerically using available program [2].

\section{Model Predictive Control for LC or ELC Systems}

Model predictive control is based on receding horizon method: for each sampling time, we solve optimization problem over finite prediction horizon, apply the first sequence of feasible optimal solution and shift the prediction horizon for the next sampling time. The procedures of model predictive control for LC or ELC systems are described as follows:

1. For each sampling time $k$, get current state $x(k)$

2. Predict the state for $N$ length of horizon using current state data $x(k)$ by recursive equation

$$
\hat{x}(k+j \mid k)=A^{j} x(k)+\sum_{i=0}^{j-1} A^{i}\left(B_{1} \hat{u}(k+j-i \mid k)+B_{2} \hat{w}(k+j-i \mid k)\right)
$$

for LC systems, or

$$
\hat{x}(k+j \mid k)=A^{j} \hat{x}(k)+\sum_{i=0}^{j-1} A^{i}\left(B_{1} \hat{u}(k+j-i \mid k)+B_{2} \hat{d}(k+j-i \mid k)\right)
$$

for ELC systems.

3. Solve the quadratic optimization:

$$
\min _{\hat{u}(k \mid k) \ldots \hat{u}(k+N-1 \mid k)}\left(\sum_{i=0}^{N-1}\left(\hat{x}(k+i \mid k)-x_{r e f}\right)^{T} Q\left(\hat{x}(k+i \mid k)-x_{r e f}\right)+\hat{u}(k+i \mid k)^{T} R \hat{u}(k+i \mid k)\right)
$$

subject to:

$$
\begin{aligned}
& \hat{x}(k+1+i \mid k)=A \hat{x}(k+i \mid k)+B_{1} \hat{u}(k+i \mid k)+B_{2} \hat{w}(k+i \mid k) \\
& \hat{v}(k+i \mid k)=E_{1} \hat{x}(k+i \mid k)+E_{2} \hat{u}(k+i \mid k)+E_{3} \hat{w}(k+i \mid k)+g_{4} \\
& 0 \leq \hat{v}(k+i \mid k) \perp \hat{w}(k+i \mid k) \geq 0, i=0,1, \ldots, N-1
\end{aligned}
$$


for LC systems, or

$$
\begin{aligned}
& \hat{x}(k+1+i \mid k)=A \hat{x}(k+i \mid k)+B_{1} \hat{u}(k+i \mid k)+B_{2} \hat{d}(k+i \mid k) \\
& E_{1} \hat{x}(k+i \mid k)+E_{2} \hat{u}(k+i \mid k)+E_{3} \hat{d}(k+i \mid k) \leq g_{4} \\
& \sum_{i=1}^{p} \prod_{j \in \phi_{i}}\left(g_{4}-E_{1} \hat{x}(k+i \mid k)-E_{2} \hat{u}(k+i \mid k)-E_{3} \hat{d}(k+i \mid k)\right)_{j}=0 \\
& i=0,1, \ldots, N-1
\end{aligned}
$$

for ELC systems, where $Q$ and $R$ are weighting matrices.

4. Apply the first optimal solution $\hat{u}^{*}(k \mid k)$ to the plant, and ignore the next solution $\hat{u}^{*}(k+1 \mid k) \ldots \hat{u}^{*}(k+N-1 \mid k)$.

5. Shift the prediction horizon for $k+1$. Go to step 1 .

As stated in Propositions 1 and 2, the optimization problem in Step 3 can be formulated as optimization problem subject to LCP/ELCP. Furthermore, as shown in Remarks 6 and 8, these problems can be solved numerically using available program. This results in on-line optimization procedures for model predictive control computation.

\section{$7 \quad$ Example}

Example 1: This example provides an application of Problem 2: the quadratic optimization subject to ELCP. For a case study, we consider traffic light control system [6], as shown in Figure 1.

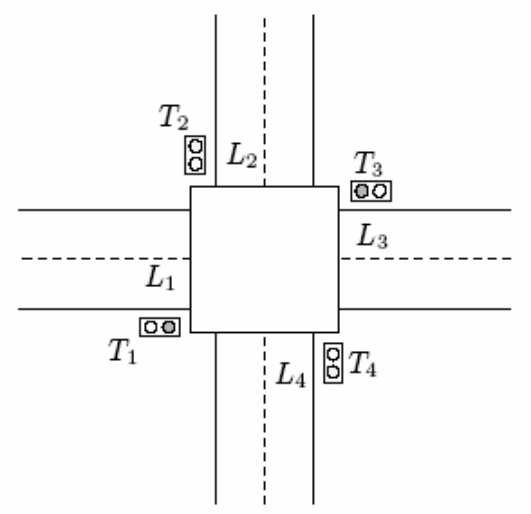

Figure 1 A traffic-light controlled system [6]. 
Table 1 The traffic-light switching scheme [6].

\begin{tabular}{|c|c|c|c|c|}
\hline Period & $T_{1}$ & $T_{2}$ & $T_{3}$ & $T_{4}$ \\
\hline$t_{0}-t_{1}$ & red & green & red & green \\
\hline$t_{1}-t_{2}$ & green & red & green & red \\
\hline$t_{2}-t_{3}$ & red & green & red & green \\
\hline$\vdots$ & $\vdots$ & $\vdots$ & $\vdots$ & $\vdots$ \\
\hline
\end{tabular}

For the sake of simplicity, we consider only the green and red light. There are four lanes $L_{1}, L_{2}, L_{3}$ and $L_{4}$. The average arrival rate in each lane is $\lambda_{i}$, and the average departure rate is $\mu_{i}$. Let $t_{0}, t_{1}, \ldots$ be the time instants at which the traffic lights switch from green to red, or vice versa, as shown in Table 1. Define $\delta_{k}=t_{k+1}-t_{k}$, and let $l_{i}(t)$ be the queue length in lane $L_{i}$ at time instant $t$. Consider lane $L_{i}$. When the light $T_{i}$ is red, the dynamics of queue is: $\dot{l}_{i}(t)=\lambda_{i}$, and when the traffic light $T_{i}$ is green, the dynamics of queue is: $\dot{l}_{i}(t)=\lambda_{i}-\mu_{i}$, if $l_{i}>0$, or $i_{i}(t)=0$, if $l_{i}=0$. Therefore we can write these equations using max expression:

$$
\begin{aligned}
& x_{2 k+1}=\max \left(x_{2 k}+b_{1} \delta_{2 k}, 0\right) \\
& x_{2 k+2}=\max \left(x_{2 k+1}+b_{2} \delta_{2 k+1}, 0\right)
\end{aligned}
$$

where:

$x_{k}=\left[\begin{array}{llll}l_{1}\left(t_{k}\right) & l_{2}\left(t_{k}\right) & l_{3}\left(t_{k}\right) & l_{4}\left(t_{k}\right)\end{array}\right]^{T}, \quad b_{1}=\left[\begin{array}{llll}\lambda_{1} & \lambda_{2}-\mu_{2} & \lambda_{3} & \lambda_{4}-\mu_{4}\end{array}\right]^{T}$, $b_{2}=\left[\begin{array}{llll}\lambda_{1}-\mu_{1} & \lambda_{2} & \lambda_{3}-\mu_{3} & \lambda_{4}\end{array}\right]^{T}$, and $k=0,1,2, \ldots$ Refer [6,7] for detail derivation.

Note that Equations (40) and (41) can be written as ELCP [6,10]: $x_{2 k+1} \geq x_{2 k}+b_{1} \delta_{2 k}, \quad x_{2 k+1} \geq 0, \quad\left(x_{2 k+1}-x_{2 k}+b_{1} \delta_{2 k}\right)_{i}\left(x_{2 k+1}\right)_{i}=0, \quad$ for $i=1 \ldots 4$. By considering $N$ horizon of switching time instants, and writing $\bar{x}=\left[x_{1}^{T}, \ldots, x_{N}^{T}\right]^{T}$ and $\bar{\delta}=\left[\delta_{0}, \ldots, \delta_{N}\right]^{T}$, we can formulate optimal control problem for the traffic-light systems for $N$ horizon as quadratic optimization subject to ELCP:

$$
\min \left(\sum_{i=0}^{\mathrm{N}-1} x_{i+1}^{T} Q x_{i}+\delta_{i}^{T} R \delta_{i}\right)
$$




$$
\begin{aligned}
& \text { subject to: } A\left[\begin{array}{l}
x^{*} \\
\delta^{*}
\end{array}\right] \geq c \\
& \sum_{j=1}^{m} \prod_{i \in \phi_{j}}\left(A\left[\begin{array}{l}
x^{*} \\
\delta^{*}
\end{array}\right]-c\right)_{i}=0
\end{aligned}
$$

Note that in [6], the authors do not use cost function in quadratic form as commonly found in optimal control literature, but rather they use worst case or the average value of queue length as cost function. Refer [6] for more detail. Using the solution of Problem 2, we can formulate this optimal control problem into MIQP. Let us take, for example, $N=4, x_{0}=[20,19,14,12]^{T}, \lambda_{1}=0.25$, $\lambda_{2}=0.12, \lambda_{3}=0.2, \lambda_{4}=0.1, \mu_{1}=0.5, \mu_{2}=0.4, \mu_{3}=0.5, \mu_{4}=0.4$, $Q=10 I_{4 \times 4}, R=0.1$. Computation using MIQP program [2] leads to optimal switching sequence $\delta^{*}=[35.9725,90.9329,30.2784,13.6832]^{T}$. Simulation result of the queue length along optimal switching scheme is shown in Figure 2.

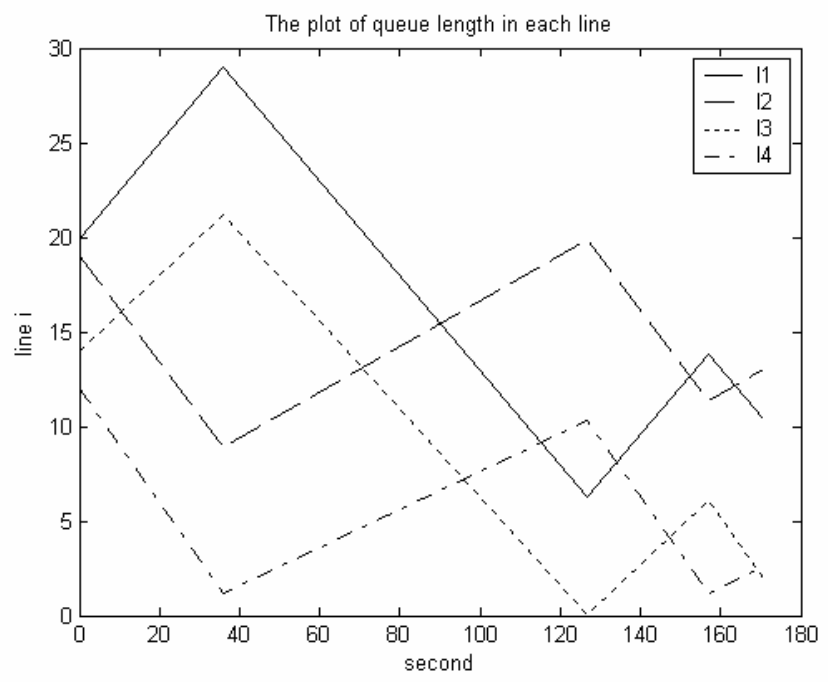

Figure 2 Plot of queue length of optimal controlled traffic-light.

Note that in $[6,7]$, since the cost function takes different form, optimization subject to ELCP gives different results.

Example 2: Consider a mechanical system consisting of inelastic stop as in Figure 3. Such system was used as a case study on modeling and wellposedness analysis of LC systems in $[10,12,16]$. 


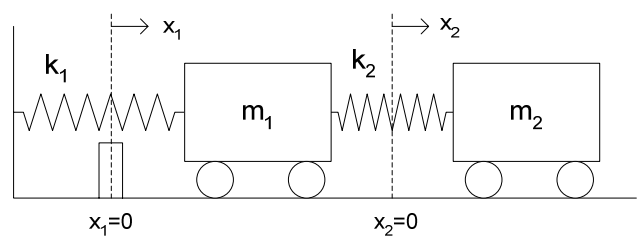

Figure 3 Mechanical systems with inelastic stop.

Let $x_{1}, x_{2}, x_{3}=\dot{x}_{1}, x_{4}=\dot{x}_{2}$ be position of cart 1 , position of cart 1 , velocity of cart 1 , and velocity of cart 2 , respectively. The discontinuous dynamics appeared when cart 1 coincide with inelastic and introduce normal force $w>0$ during $x_{1}=0$. Therefore, the dynamics of cart 1 contain discontinuity as

$$
m_{1} \ddot{x}_{1}=\left\{\begin{array}{cc}
-k_{1} x_{1}+k_{2}\left(x_{2}-x_{1}\right)_{1} & \text { if } x_{1}>0 \\
k_{2} x_{2}+w & \text { if } x_{1}=0
\end{array}\right.
$$

whereas the dynamics of cart 2 is given by

$$
m_{2} \ddot{x}_{2}=-k_{2}\left(x_{2}-x_{1}\right)
$$

By considering $v=x_{1}$ and $w$ as a complementarity variable and assuming that the mass of cart 1 and cart 2 are $1 \mathrm{~kg}$, the system can be modeled as LC system as follows

$$
\begin{aligned}
& {\left[\begin{array}{l}
\dot{x}_{1}(t) \\
\dot{x}_{2}(t) \\
\dot{x}_{3}(t) \\
\dot{x}_{4}(t)
\end{array}\right]=\left[\begin{array}{cccc}
0 & 0 & 1 & 0 \\
0 & 0 & 0 & 1 \\
-2 & 1 & 0 & 0 \\
1 & -1 & 0 & 0
\end{array}\right]\left[\begin{array}{l}
x_{1}(t) \\
x_{2}(t) \\
x_{3}(t) \\
x_{4}(t)
\end{array}\right]+\left[\begin{array}{l}
0 \\
0 \\
1 \\
0
\end{array}\right] w(t)} \\
& v(t)=\left[\begin{array}{llll}
1 & 0 & 0 & 0
\end{array}\right]\left[\begin{array}{l}
x_{1}(t) \\
x_{2}(t) \\
x_{3}(t) \\
x_{4}(t)
\end{array}\right], \\
& 0 \leq v(t) \perp w(t) \geq 0
\end{aligned}
$$

The simulation of open-loop systems is obtained by approximating the continuous-time LC systems by discrete-time model using backward Euler method and formulating trajectory solution computation as a solution of LCP [10] as shown in Figure 4. 


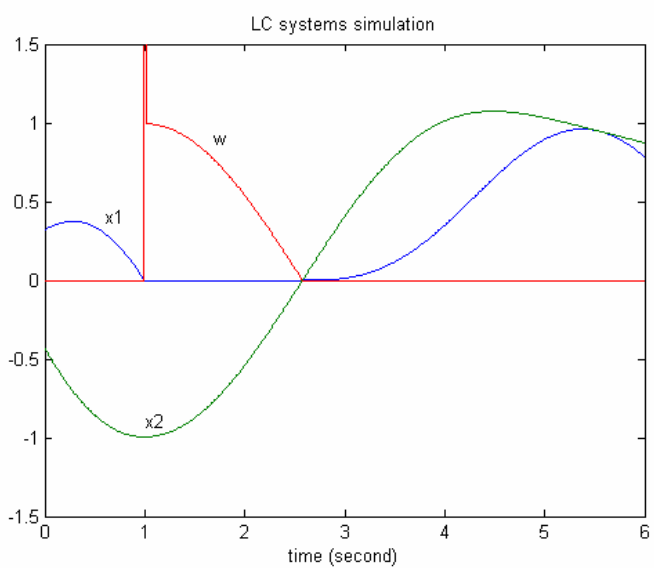

Figure 4 Open loop response of LC systems.

For the purpose of control synthesis, we introduce external force on both cart as $u=\left[\begin{array}{ll}F_{1} & F_{2}\end{array}\right]^{T}$, which leads to modified LC model:

$$
\begin{aligned}
& {\left[\begin{array}{l}
\dot{x}_{1}(t) \\
\dot{x}_{2}(t) \\
\dot{x}_{3}(t) \\
\dot{x}_{4}(t)
\end{array}\right]=\left[\begin{array}{cccc}
0 & 0 & 1 & 0 \\
0 & 0 & 0 & 1 \\
-2 & 1 & 0 & 0 \\
1 & -1 & 0 & 0
\end{array}\right]\left[\begin{array}{l}
x_{1}(t) \\
x_{2}(t) \\
x_{3}(t) \\
x_{4}(t)
\end{array}\right]+\left[\begin{array}{cc}
0 & 0 \\
0 & 0 \\
-1 & 0 \\
0 & -1
\end{array}\right] u(k)+\left[\begin{array}{l}
0 \\
0 \\
1 \\
0
\end{array}\right] w(t)} \\
& v(t)=\left[\begin{array}{llll}
1 & 0 & 0 & 0
\end{array}\right]\left[\begin{array}{l}
x_{1}(t) \\
x_{2}(t) \\
x_{3}(t) \\
x_{4}(t)
\end{array}\right] \\
& 0 \leq v(t) \perp w(t) \geq 0
\end{aligned}
$$

The model predictive control solution is obtained using method previously developed in Section 6. Approximating the discrete-time LC model and choosing $Q=10 I_{4 \times 4}, R=0.1 I_{2 \times 2}$, and $N=3$, resulted in control system response shown in Figure 5.

As far as the authors concern, the plant considered here has not been studied in hybrid control synthesis, but rather in analysis of well-posedness properties of hybrid systems $[10,12,16]$. 


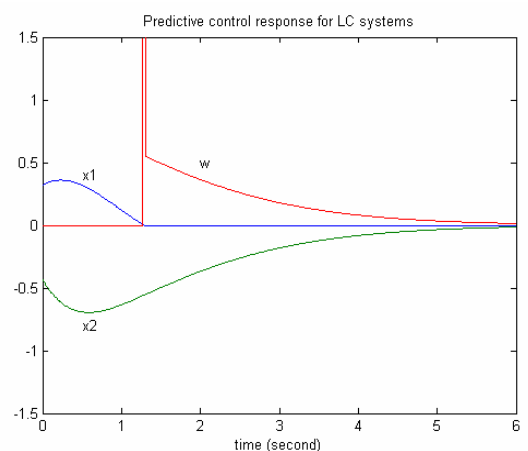

(a)
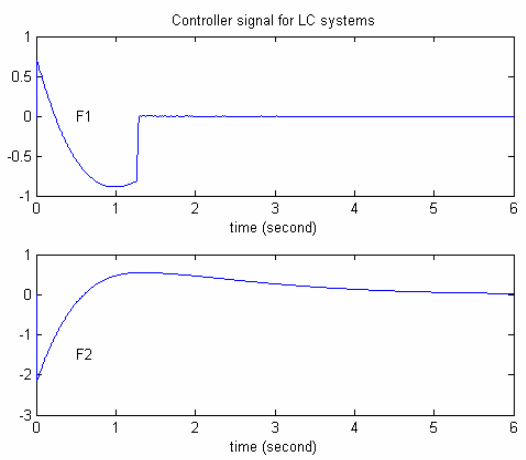

(b)

Figure 5 (a) Predictive control response for LC systems (b) Controller signal.

Example 3: Consider two tank systems in Figure 6, which is adopted from [14].

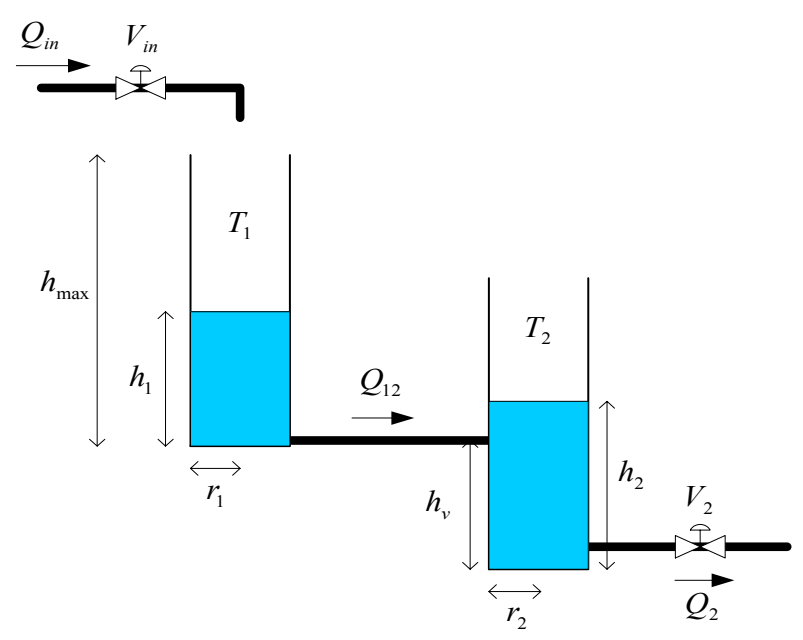

Figure 6 Two tank system.

Let $h_{1}$ and $h_{2}$ be the height of fluid level inside tank 1 and 2, respectively. Both valves $V_{\text {in }}$ and $V_{2}$ are continuous and have range of $0 \% \leq V_{\text {in }} \leq 100 \%$, $0 \% \leq V_{2} \leq 100 \%$. The dynamics of the tank systems is given by differential equations $\quad d h_{1} / d t=\left(Q_{\text {in }}-Q_{12}\right) / A_{1}$ and $d h_{2} / d t=\left(Q_{12}-Q_{2}\right) / A_{2}$ where discontinuity arises as $Q_{12}=\left\{\begin{array}{ll}k_{12} \sqrt{h_{1}-\left(h_{2}-H\right)} & \text { if } h_{2}>h_{v} \\ k_{12} \sqrt{h_{2}} & \text { if } h_{2} \leq h_{v}\end{array}\right.$. 
By writing $T_{A 1}=T_{s} / A_{1}, T_{A 2}=T_{s} / A_{2}$, discretizing the differential equations using Euler approximation, and linearizing the square-root terms, we obtain Piece-wise Affine model:

$$
\begin{aligned}
& h_{1}(k+1)=\left\{\begin{array}{l}
h_{1}(k)+T_{A 1}\left(V_{i n}(k) Q_{\max }-\bar{k}_{1}\left(h_{1}(k)-h_{2}(k)+h_{v}\right)\right), \text { if } h_{2}(k)>h_{v} \\
h_{1}(k)+T_{A 1}\left(V_{i n}(k) Q_{\max }-\bar{k}_{1} h_{1}(k)\right),
\end{array}\right. \\
& h_{2}(k+1)= \begin{cases}h_{2}(k)+T_{A 2}\left(\bar{k}_{1}\left(h_{1}(k)-h_{2}(k)+h_{v}\right)-\bar{k}_{2}\left(V_{2}(k)+h_{2}(k)-1\right)\right), \text { if } h_{2}(k)>h_{v} \\
h_{2}(k)+T_{A 2}\left(\bar{k}_{1} h_{1}(k)-\bar{k}_{2}\left(V_{2}(k)+h_{2}(k)-1\right)\right) & \text {, if } h_{2}(k) \leq h_{v}\end{cases}
\end{aligned}
$$

Moreover, we can describe the above equation using max term as:

$$
\begin{aligned}
& h_{1}(k+1)=\left(1-T_{A 1} k_{12}\right) h_{1}(k)+T_{A 1} Q_{\max } V_{i n}(k)+T_{A 1} k_{12} \max \left(h_{2}(k)-h_{v}, 0\right) \\
& h_{2}(k+1)=T_{A 2} k_{12} h_{1}(k)+\left(1-T_{A 2} k_{2}\right) h_{2}(k)-T_{A 2} k_{2} V_{2}(k)-T_{A 2} \max \left(k_{12} h_{2}(k)-k_{12} h_{v}-k_{2},-k_{2}\right)
\end{aligned}
$$

By introducing the auxiliary variables $d_{1}, d_{2} \in R, d_{1}(k)=\max \left(h_{2}(k)-h_{v}, 0\right)$ and $d_{2}(k)=\max \left(k_{12} h_{2}(k)-k_{12} h_{v}-k_{2},-k_{2}\right)$, we can write the ELC form: $d_{1}(k)-h_{2}(k)+h_{v} \geq 0, \quad d_{1}(k) \geq 0, \quad\left(d_{1}(k)-h_{2}(k)+h_{v}\right) d_{1}(k)=0, \quad$ and, $d_{2}(k)-k_{12} h_{2}(k)+k_{12} h_{v}+k_{2} \geq 0, \quad d_{2}(k)+k_{2} \geq 0$, $\left(d_{2}(k)-k_{12} h_{2}(k)+k_{12} h_{v}+k_{2}\right)\left(d_{2}(k)+k_{2}\right)=0$.

Collecting the above terms, we obtain ELC model:

$$
\begin{aligned}
& {\left[\begin{array}{l}
h_{1}(k+1) \\
h_{2}(k+1)
\end{array}\right]=\left[\begin{array}{cc}
\left(1-T_{A 1} k_{12}\right) & 0 \\
T_{A 2} k_{12} & \left(1-T_{A 2} k_{2}\right)
\end{array}\right]\left[\begin{array}{l}
h_{1}(k+1) \\
h_{2}(k+1)
\end{array}\right]+\left[\begin{array}{cc}
T_{A 1} Q_{\max } & 0 \\
0 & -T_{A 2} k_{2}
\end{array}\right]\left[\begin{array}{l}
V_{i n} \\
V_{2}
\end{array}\right]+\left[\begin{array}{cc}
T_{A 1} k_{12} & 0 \\
0 & -T_{A 2}
\end{array}\right]\left[\begin{array}{l}
d_{1}(k) \\
d_{2}(k)
\end{array}\right]}
\end{aligned}
$$

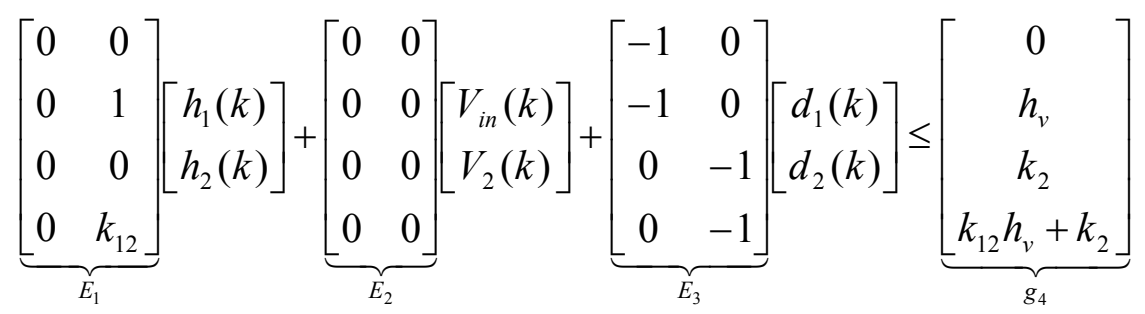

$$
\begin{aligned}
& d_{1}(k)\left(d_{1}(k)-h_{2}(k)+h_{v}\right)+\left(d_{2}(k)+k_{2}\right)\left(d_{2}(k)-k_{12} h_{2}(k)+k_{12} h_{v}+k_{2}\right)=0
\end{aligned}
$$

We apply the predictive control method developed in Section 6. Choose weighting matrices $Q=100 I_{2 \times 2}, R=0.01 I_{2 \times 2}$. The simulation results for reference tracking response are shown in Figure 7. 


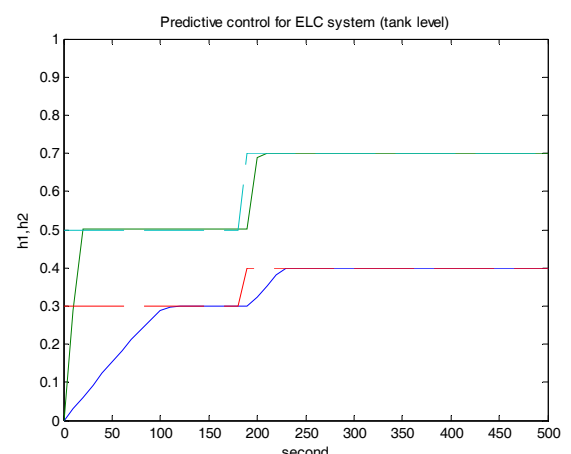

(a)
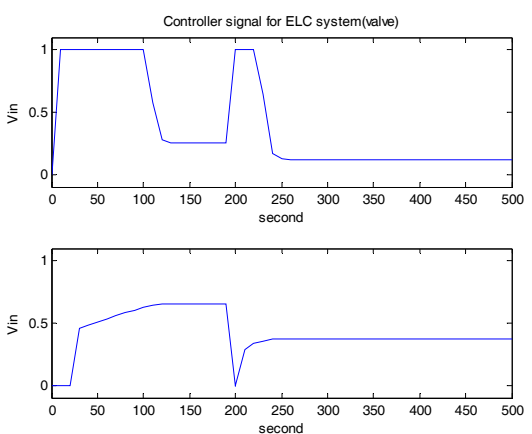

(b)

Figure 7 Predictive control response for set point tracking.

Note that in [14], the two tank system consists of discrete valve that has only two states: ON or OFF. The controller used sequential logic strategy and was not intended to track the level to desired set point, but rather to maintain the level on the specific range.

\section{Conclusion}

In this paper, model predictive control scheme for LC and ELC systems was proposed. The quadratic optimization problem along prediction horizon is recast as mixed integer quadratic program (MIQP), which can be computed numerically using MIQP solver. As demonstrated by the simulation results on traffic light control system, the proposed method on solving quadratic optimization subject to ELCP generates optimized switching time of trafficlight control system. Two other control simulations for mechanical system with inelastic stop and two tank systems also show that the proposed predictive control gives satisfactory set point tracking results, both for LC and ELC dynamical systems.

\section{References}

1. Belegundhu, A. D., Chandrupatla, T. R., Optimization concepts and application in engineering, Prentice-Hall (1999).

2. Bemporad, A., Mignone, D., Miqp.m: a matlab function for solving mixed integer quadratic programs. ETH Zurich, Switzerland (2000). http://www.dii.unisi.it/ hybrid/tools/miqp/

3. Bemporad, A., Morari, M., Control of systems integerating logic, dynamics and constraints, Automatica, Vol. 35, No. 3, March (1999). 
4. Brogliato, B., Some perspectives on the analysis and control of complementarity systems, IEEE Transactions on Automatic Control, Vol. 48, No. 4, 918-935, June (2003).

5. De Schutter, B., De Moor, B., The extended linear complementarity problems, Mathematical Programming, 71(3), December (1995).

6. De Schutter, B., De Moor, B., The extended linear complementarity problem and its application in the analysis and control of discrete event systems and hybrid systems, in Proceedings of the IEEE Singapore International Symposium on Control Theory and Applications (SISCTA 97), Singapore, pp. 394-398 (1997).

7. De Schutter, B., De Moor, B., Optimal traffic light control for a single intersection, European Journal of Control, vol. 4, no. 3, pp. 260-276, 1998.

8. De Schutter, B., Heemels, W. P. M. H., Bemporad, A., On the equivalence of linear complementarity problems, Operations Research Letters, 30, 211$222(2002)$.

9. Ferris, M. C., Munson, T. S., Complementary problems in GAMS and PATH solver, Mathematical Programming Technical Report, September (1998). http://www.cs.wisc.edu/cpnet/

10. Heemels, W. P. M. H., Linear complementarity systems: a study in hybrid dynamics, PhD Dissertation, Dept. of Electrical Engineering, Eindhoven University of Technology, The Netherlands (1999).

11. Heemels, W. P. M. H., De Schutter, B., Bemporad, A., Equivalence of hybrid dynamical models, Automatica, 37, pp. 1085-1091 (2001).

12. Heemels, W. P. M. H., Schumacher, J. M., Weiland, S., Linear complementarity systems, SIAM Journal of Applied Mathematics, Vol. 294, 1234-1269 (2000).

13. IEEE Transactions on Automatic Control, Vol. 43, No. 4, April (1998).

14. Kowalewski, S., Stursberg, O., Fritz, M., Graf, H., Hoffmann, I., Preußig, J., Remelhe, M., Simon, S., Treseler, H., A case study in control-aided analysis and discretely controlled continuous systems: the two tanks problems, presented in $5^{\text {th }}$ International Workshop on Hybrid Systems, Notre Dame, USA (1997).

15. Proceedings of the IEEE, Vol.88, No. 7, July (2000).

16. van der Schaft, A. J., Schumacher, J. M., The complementary-slackness class of hybrid systems, Mathematics of Control, Signals and Systems, No. 9, 266-301 (1996).

17. van der Schaft, A. J., Schumacher, J. M., Complementarity modeling of hybrid systems, IEEE Transactions on Automatic Control, Vol. 43, No. 4, 483-490, April (1998). 